\title{
CONCEPCIONES Y PRÁCTICAS DE AULA DE PROFESORES DE CIENCIAS, EN FORMACION INICIAL DE PRIMARIA Y SECUNDARIA
}

MELLADO JIMÉNEZ, $\mathrm{V}$. Departamento de Didáctica de Ias Ciencias Experimentales y de las Matemáticas. Facultad de Educación. Universidad de Extremadura. Av. Elvas, s/n. 06071 Badajoz.

\begin{abstract}
SUMMARY
The present article describes a research carried out with four student teachers of primary and secondary science education. The preservice teachers' conceptions of the nature of science and learning and teaching of science were analyzed and compared with their classroom practice when teaching science lessons. Transfer of the conceptions to the classroom was not immediate and there was influence from other factors. Lastly the implications of the research for teacher education are discussed.
\end{abstract}

\section{INTRODUCCIÓN}

El profesor es un factor clave que determina el éxito o el fracaso de cualquier innovación curricular (Mitchener y Anderson, 1989) y, aunque la investigación en didáctica de las ciencias se ha centrado fundamentalmente en problemas relativos al alumno y al aprendizaje, a partir de la mitad de la década de los ochenta se detecta un aumento de las investigaciones que tienen como protagonistas a los profesores y profesoras de ciencias (Furió, 1994).

Desde la didáctica general, el paradigma del «pensamiento del profesor» ha aportado numerosos resultados sobre el pensamiento y la práctica de los profesores. El profesor no es un técnico que aplica instrucciones, sino un constractivista que procesa información, toma decisiones, genera rutinas y conocimiento práctico, y posee creencias que influyen en su actividad profesional (Marcelo, 1987 y 1994). En los últimos años, el paradigma deI pensamiento del profesor evoluciona hacia un mayor compromiso con los contenidos (Anderson y Mitchener, 1994; Marcelo, 1993). Las estrategias didácticas de los profesores son muy diferentes según la materia que enseñan, y sus activiđades y prácticas pedagógicas no son fijas, sino que dependen de la asignatura (Stodolsky, 1991). Cada materia tiene unas tradiciones y creencias, a menudo implícitas, sobre la mejor manera de enseñarla y aprenderla, que se transmiten por los especialistas a los profesores en formación.

Shulman (1986 y 1993) considera que, además del conocimiento de la materia y del conocimiento psicopedagógico general, los profesores desarrollan un conocimiento específico sobre la forma de enseñar su materia, que denomina el conocimiento didáctico del contenido. EI profesor es el mediador que transforma el contenido en representaciones comprensibles a los alumnos.

El conocimiento de la materia que tienen los profesores de ciencias influye para que desarrollen una enseñanza más eficaz (Thomas y Gilbert, 1989; Duschl y Wright, 1989; Carlsen, 1993; Lee y Porter, 1993). Sin embargo, el conocimiento de la materia para un profesor de ciencias es distinto que para un especialista, ya que el conocimiento del contenido de los profesores de ciencias está relacionado con el propio proceso de la enseñanza (Anderson, 1989; Hauslein et al., 1992; Pomeroy, 
1993; Gess-Newsome y Lederman, 1993; Lederman et al., 1994).

Por otra parte, y desde una perspectiva constructivista (Hewson y Hewson, 1989), se considera que los profesores de ciencias tienen concepciones sobre la ciencia y sobre la forma de aprenderla y enseñarla, fruto de sus años de escolaridad, que están profundamente arraigadas. Aunque el término concepción o creencia educativa de los profesores se ha utilizado en la investigación con distintos matices, la creencia o concepción implica una convicción o valoración sobre algo (Koballa y Crawley 1985 ) y en ellas juega un importante papel la viabilidad, la componente social y la predisposición para actuar (Tobin et al., 1994). El estudio de las concepciones de los profesores de ciencias cobra así una especial importancia, como un primer paso pará generar en los propios profesores unas concepciones y prácticas más adecuadas (Gil, 1993; Hewson, 1993).

En España, como consecuencia del desarrollo de la LOGSE, confluirán en los centros de secundaria en los dos primeros años de la educación secundaria obligatoria en el área de ciencias de la naturaleza, maestros especialistas en ciencias y licenciados en ciencias, dos colectivos de profesores con tradiciones formativas y de organización muy diferentes. Uno de los objetivos de nuestra investigación (Mellado, 1995a) es conocer las concepciones sobre la ciencia y sobre la enseñanza y aprendizaje de las ciencias de una muestra de ambos colectivos de profesores, al final de su etapa de forma* ción universitaria, y compararlas con sus conductas docentes al impartir una lección de ciencias.

\section{ANTECEDENTFS}

\section{Concepciones de los profesores sobre la naturaleza de la ciencia}

En una revisión sobre el tema, Lederman (1992) señala que desde la década de los cincuenta existen investigaciones que abordan desde una perspectiva "procesoproducto» las concepciones de los profesores sobre la naturaleza de la ciencia. Estas primeras investigaciones asumen que las concepciones de los profesores sobre la naturaleza de la ciencia afectan a las concepciones de los estudiantes e influyen en la conducta de los profesores en el aula y en el ambiente de clase. Una conclusión general de estos primeros trabajos es que la mayoría de los profesores analizados de primaria y secundaria no poseen puntos de vista adecuados sobre la naturaleza de la ciencia; otra conclusión es que los antecedentes aca* démicos de los profesores no están significativamente relacionados con sus concepciones sobre la naturaleza de la ciencia.

En los últimos años ha aumentado el estudio de las concepciones de los profesores sobre la naturaleza de la ciencia. Muchas investigaciones encuadran a la mayoría de los profesores de ciencias en alguna de las formas del positivismo (Abell y Smith, 1994; Aguirre et al., 1990; Ballenilla, 1992; Currais y Pérez, 1994; Duschl y Wright, 1989; Porlán, 1989; Powell, 1994; Rubba y Harkness, 1993; Ruggieri et al., 1993). Sin embargo, hay resultados discrepantes que nos hacen pensar que la situación es más compleja. los trabajos de Acevedo (1994) y de Lakin y Wellington (1994) muestran que, atunque los profesores de ciencias tienen rasgos empiristas, no pueden encuadrarse de una forma simpista en un inductivismo ingenuo, sino que su posición es más compleja. Koulaidis y Ogborn (1989) señalan que los profesores de ciencias asumen posiciones más próximas al contextualismo de Kuhn (1971) que al empirismo; también destacan que un alto porcentaje de los profesores posee un punto de vista ecléctico sobre la naturaleza de la ciencia, y sus concepciones no pueden considerarse consistentemente asociadas con una orientación filosófica particular.

Una de las causas de la discrepancia en los resultados de las investigaciones puede encontrarse en la disparidad de las metodologías utilizadas y la distinta valoración filosofica que hacen los investigadores de los propios instrumentos metodológicos utilizados (Koulaidis y Ogborn, 1995). Inicialmente la mayoría de las investigaciones sobre las concepciones epistemológicas de los profesores de ciencias utilizan el cuestionario. Sin cmbargo, Lederman y O'Malley (1990) consideran que los cuestionarios sobre la naturaleza de la ciencia dan resultados simplificados que no se corresponden con las orientaciones más ricas manifestadas en la entrevistas, por eso abogan por la utilización de métodos cualitativos de investigación. En los últimos años es creciente el empleo de metodologías cualitativas y de estudios de caso gue nos aportan un cuadro más completo de la situación (Blanco, 1991; Marcelo y Parrilla, 1991).

Existe también una coincidencia casi generaliyadà entre los investigadores en destacar que la filosofía de la ciencia no se incluye en los programas de formación del profesorado de ciencias, y que debería abordarse ayudando a los profesores en formación a reflexionar sobre sus propias concepciones epistemológicas. El profesor de ciencias tiene que tratar los aspectos de filosofía e historia de la ciencia, relacionándolos con su propia actividad de enseñanza de las ciencias (Mellado y Carracedo, 1993). Destacamos en este sentido dos trabajos que incorporan a la formación de profesores la historia de la ciencia (Moreno, 1995) y la reflexión epistemológica (Jiménez, 1995).

\section{Concepciones de los profesores sobre la enseñanza y aprendizaje de las ciencias}

Cuando los profesores comienzan sus cursos de formación ya tienen organizada su estructura de creencias sobre la enseñanza y aprendizaje de las ciencias (Shaw y Cronin-Jones, 1989), y los años de escolaridad que los profesores de ciencias han pasado como alumnos tienen una gran influencia en sus concepciones pedagógicas (Briscoe, 1991; Gunstone et al., 1993; Hewson y Hew- 
son, 1989; Wallace y Louden, 1992; Young y Kellogg 1993). Además las creencias pedagógicas son muy estables y apenas cambian durante el programa de formación inicial (Aguirre y Haggerty, 1995; Marcelo, 1995).

En relación con investigaciones cuantitativas de las creencias de los profesores sobre el aprendizaje de Ias ciencias, Spear (1984) indica que los profesores de secundaria ingleses tienen mayores expectativas sobre el potencial hacia la ciencia de los alumnos que de las alumnas, lo cual condiciona la evaluación. Porlán (1989) encuentra que entre los profesores de primaria en formación predomina el modelo implícito de «mente en blanco» para el aprendizaje de las ciencias. En cambio, para Aguirre y otros (1990), aunque más del $40 \%$ de Ios profesores de secundaria en formación inicial tiene una idea del aprendizaje de la ciencia como una recepción de conocimiento por los alumnos, un $27 \%$ considera el aprendizaje como un cambio del conocimiento que tiene el alumno.

En cuanto a la enseñanza de las ciencias, para Porlán (1989), los profesores de primaria en formacion rechazan el método tradicional expositivo y caen en un prácticismo espontaneista. En cambio, Aguirre y otros (1990), encuentran que las concepciones de los profesores de secundaria canadienses en formación inicial sobre la enseñanza de las ciencias están divididas entre la transmisión de conocimiento y una guía para la actividad del alumno. Para Pomeroy (1993), Ios profesores tienen una concepción más tradicional que las profesoras y los profesores de secundaria significativamente más tradicionales que los de primaria.

Otros trabajos utilizan una metodología cualitativa y también asignan a los profesores concepciones tradicionales sobre la enseñanza y aprendizaje de las ciencias (Ballenilla, 1992; Gunstone et al., 1993; Smith y Neale, 1991). En cambio, en el trabajo de López (1994), distingue entre los profesores que tienen una concepción "constructivista compleja», en la que las ideas de los alumnos son modos alternativos de interpretar el mundo, y los que tienen una concepción «constructivista simplificada», por la que conciben el conocimiento de los alumnos como errores que hay que eliminar a través de Ia enseñanza de las ciencias.

Otro aspecto es la relación entre las concepciones de los profesores sobre la enseñanza y aprendizaje de las ciencias y sus concepciones sobre la naturaleza de las ciencias. Pomeroy (1993) y Koulaidis y Ogborn (1995) establecen explícitamente una correspondencia parcial entre ellas, aunque reconocen algunas contradicciones. Sin embargo, Powell (1994) senala disparidad entre las concepciones sobre la ciencia de un profesor y las que tiene sobre la enseñanza y el aprendizaje de las ciencias.

Investigaciones sobre la relación entre las concepciones de los profesores de ciencias y la práctica del aula

En este apartado hay notables diferencias entre las conclusiones de las investigaciones realizadas. Existen tra- bajos que muestran una correspondencia entre las concepciones de los profesores sobre la naturaleza de la ciencia y su conducta docente en el aula (Ballenilla, 1992; Brickhouse, 1990; Cachapuz, 1994; Gallagher, 1991). Sin embargo, otras investigaciones (Benson, 1989; Duschl y Wright, 1989; Lederman, 1986; Lederman y Zeidler, 1987) no encuentran relación entre las concepciones sobre la naturaleza de la ciencia de los profesores y su comportamiento en el aula. En lo que hay acuerdo entre los investigadores, según señala Iederman (1992), es que la posible influencia de las concepciones de los profesores sobre la naturaleza de la ciencia en la práctica del aula está mediatizada por otra serie de complejos factores, tales como las restricciones del currículo, las políticas administrativas, Ias actitudes de los profesores sobre los estudiantes y el aprendizaje, etc.

Los trabajos de Tobin y Espinet (1989), Mitchener y Anderson (1989), Cronin-Jones (1991), Lorsbach y otros (1992) y Dillon y otros (1994) defienden la relación entre las concepciones de los profesores sobre la enseñanza y aprendizaje de las ciencias y la conducta en el aula al enseñar ciencias.

Sin embargo otras investigaciones matizan los resultados anteriores. López ( 1994 ) sólo encuentra una relación parcial entre las concepciones de los profesores de primaria sobre la enseñanza y el aprendizaje de las ciencias y la práctica del aula. Huibregtse y otros (1994) encuentran contradicciones entre las ideas conscientes y las menos conscientes que son mediatizadas por el conjunto de valores, sentimientos, pensamientos y acciones formadas durante sus experiencias como estudiantes y reconocen que las concepciones y opiniones expresadas por los profesores no necesariamente predicen sus conductas en el aula. Para Freire y Chorao (1992), Ios principios prácticos de actuación tienen características comunes en la mayoría de los profesores de fúsica de secundaria estudiados, independientemente de sus creencias. En la complejidad del aula, Ios profesores construyen modelos simplificados que les permiten actuar y que les resultan cómodos y no conflictivos (Lee y Porter, 1993; Wallace y Louden, 1992). La transferencia de las concepciones de los profesores de ciencias a la práctica del aula puede no producirse (Gess-Newsome y Lederman, 1993) si Ios profesores carecen de esquemas de acción prácticos coherentes con sus creencias (Tobin, 1993).

\section{METODOLOGÍA}

El objetivo de nuestra investigación es conocer las concepciones sobre la naturaleza de Ia ciencia y la didáctica de las ciencias, así como su relación con la conducta en el aula al impartir una lección de ciencias, de una muestra de maestros de la especialidad de ciencias y de licenciados en ciencias. Para ello realizamos un estudio de caso de cuatro profesores al final de su etapa de formación inicial, durante el curso 1992-93. Dos de los profesores son maestros especialistas en ciencias, du- 
rante el tercer año de la Diplomatura de Magisterio, y dos son licenciados en ciencias, uno de física y otro de biología, durante la realización del Certificado de Aptitud Pedagógica.

Para la sclección de participantes, en primer lugar se informó de la investigación a los maestros y licenciados que durante el curso 1992-93 realizaron los estudios anteriormente señalados en la Universidad de Extremadura, en Badajoz. Una primera muestra la formaron doce profesores en formación que voluntariamente deseaban participar en la investigación. Para la selección final de cuatro participantes se tuvo en cuenta que hubiese dos maestros y dos licenciados de distinta materia (física y biología), los cuales hubiesen completado adecuadamente los protocolos de recogida de datos, el curso en que realizaron las prácticas de enseñanza y que tuviesen una alta expresividad y motivación en la producción de datos cualitativos.

En cuanto a los procedimientos de recogida y análisis de datos, durante años ha existido una fuerte polémica entre las dos tradiciones de investigación en educación, que de una manera reduccionista podríamos decir que enfrentaba los métodos cuantitativos y los cualitativos. Hoy esta dicotomía está superada para muchos autores (Woods, 1987; Estebaranz, 1992), y a menudo se emplea una combinación de métodos variados (Marcelo, 1992). El estudio de caso como eel examen de un ejemplo en acción» (Walker, 1983) está cobrando una especial importancia en la investigación sobre la formación del profesorado (Blanco, 1991). EI estudio de caso nos permite profundizar más en el pensamiento y la acción de un número reducido de personas. Nuestra pretensión no es que los datos sean generalizables, sino que utilizando Ios criterios de fiabilidad y validez de la investigación naturalista, etnográfica o cualitativa (Goetz y Lecompte, 1988; Marcelo y Parrilla, 1991) podamos obtener resultados que puedan ser comparados con los de otras investigaciones y que nos permitan avanzar en la mejora de la enseñanza de las ciencias.

Los procedimientos de recogida de datos que hemos utilizado (Fig. I) son el cuestionario INPECIP elaborado por Porlán (1989), entrevistas semiestructuradas grabadas en audio, documentos personales, observaciones de aula durante la realización de las prácticas de enseñanza grabadas en vídeo y, finalmente, entrevistas de estimulación del recuerdo realizadas simultáneamente al visionado del vídeo. Previamente todos los sujetos participaron en una sesión de microenseñanza que fue grabađa en vídeo, el objetivo de la cual era obtener datos de cada uno de los participantes para elaborar un gutón individualizado para la entrevista inicial, así como realizar un trabajo previo de campo que ayudase a compartir significados. No incluimos los diarios de prácticas, porque en investigaciones precedentes (Mellado y Bermejo, 1995) observamos que los diarios se centran en cuestiones generales y no en problemas específicos de enseñanza y aprendizaje de las ciencias.

Los cuatro participantes, a los que denominamos con los nombres supuestos de David (licenciado en Física),
Miguel (licenciado en Biología), Ana (maestra especialista en Ciencias) y Julio (maestro especialista en Ciencias), realizaron las prácticas de enseñanza en centros de la provincia de Badajoz. Las grabaciones de la observación de clase fueron realizadas en los siguientes cursos y asignaturas: David en la asignatura de Física y Química de $2^{\circ}$ de BUP, Miguel en la asignatura de Ciencias Naturales de $1^{\circ}$ de BUP, Ana en Ciencias de la Naturaleza de $6^{\circ}$ de EGB, y Julio en Ciencias de la Naturaleza de $8^{\circ}$ de EGB. I a observación de clase se realizó sobre el tema «Energía y medio ambiente», que al ser interdisciplinar permitió a cada participante darle una crientación específica según su propia formación y el nivel de la clase.

El análisis del cuestionario y de la entrevista inicial se realizó por medio de mapas cognitivos. Los mapas conceptuales fueron inicialmente desarrollados por Novak y Gowin (1988) y han sido ampliamente validados y utilizados en didáctica de las ciencias (GessNewsome y Lederman, 1993; González, 1992; I.ópez Rupérez, 1991; Ontoria et al., 1992). Llinares (1992) da a los mapas una orientación distinta y los utiliza como mapas cognitivos para representar gráficamente la estructura de creencias de profesores en formación sobre la enseñanza de las matemáticas.

Los mapas cognitivos relacionan, de una forma parcialmente jerarquizada, unidades de información con un sentido más amplio que los conceptos utilizados en los mapas conceptuales. La representación por medio de mapas cognitivos permite una visión global y no fragmentada de las creencias de los profesores sobre la ciencia y la enseñanza de las ciencias. Para la construcción de los mapas cognitivos de un profesor a partir del cuestionario clasificamos las respuestas por categorías. A continuación en cada grupo de respuestas se enlazan las frases de los ítems de las más generales e inclusoras a las más particulares, formando un mapa cognitivo de creencias, realizado con una técnica análoga a la que utilizan Novak y Gowin (1988) para los conceptos. Para la construcción del mapa a través de la entrevista, se codifica cada frase que suponga una unidad de información, posteriormente se clasifican en categorías y se relacionan gráficamente en forma de mapa cognitivo. Por ejemplo, la pregunta 163 realizada a David se clasifica en once unidades de información:

\section{D.163: ¿Qué importancia le das a la explicación del profesor?}

Respuesta de David: [La explicación es inevitable porque hay que decir al alumno cosas que no sabe]'. [El que las sabe es el profesor y tiene que transmitirlas] ${ }^{2}$. Por tanto [la exposicion de los contenidos es inevitable $]^{3}$, pero pienso que [hay profesores, y eso se ve en los centros de secundaria, que llegan: «Tema tal, bla, bla, bla...», y el alumno a copiar todo el tiempo. Y eso no es. Se trata de darle los conceptos que quiera $]^{4}$ [no dictarle, sino explicarle el concepto y darle vuelta, explicărselo de varias formas, buscarle ejemplos] $]^{3}$. Porque también resultaría muy fácil tlegar y decir: «Tema tal. Principios de la dinámica. Dinámica: es la parte de la física que bla, bla, bla...", y el alumno copiando. Sería lo más sencillo del mundo, pero eso a mí no me sirve de nada, porque Ino se trata de que yo Io explique sino de que ellos lo entiendan] ${ }^{6}$. [Tengo que buscar de 
Figura 1

Procedimientos de recogida y análisis de datos.

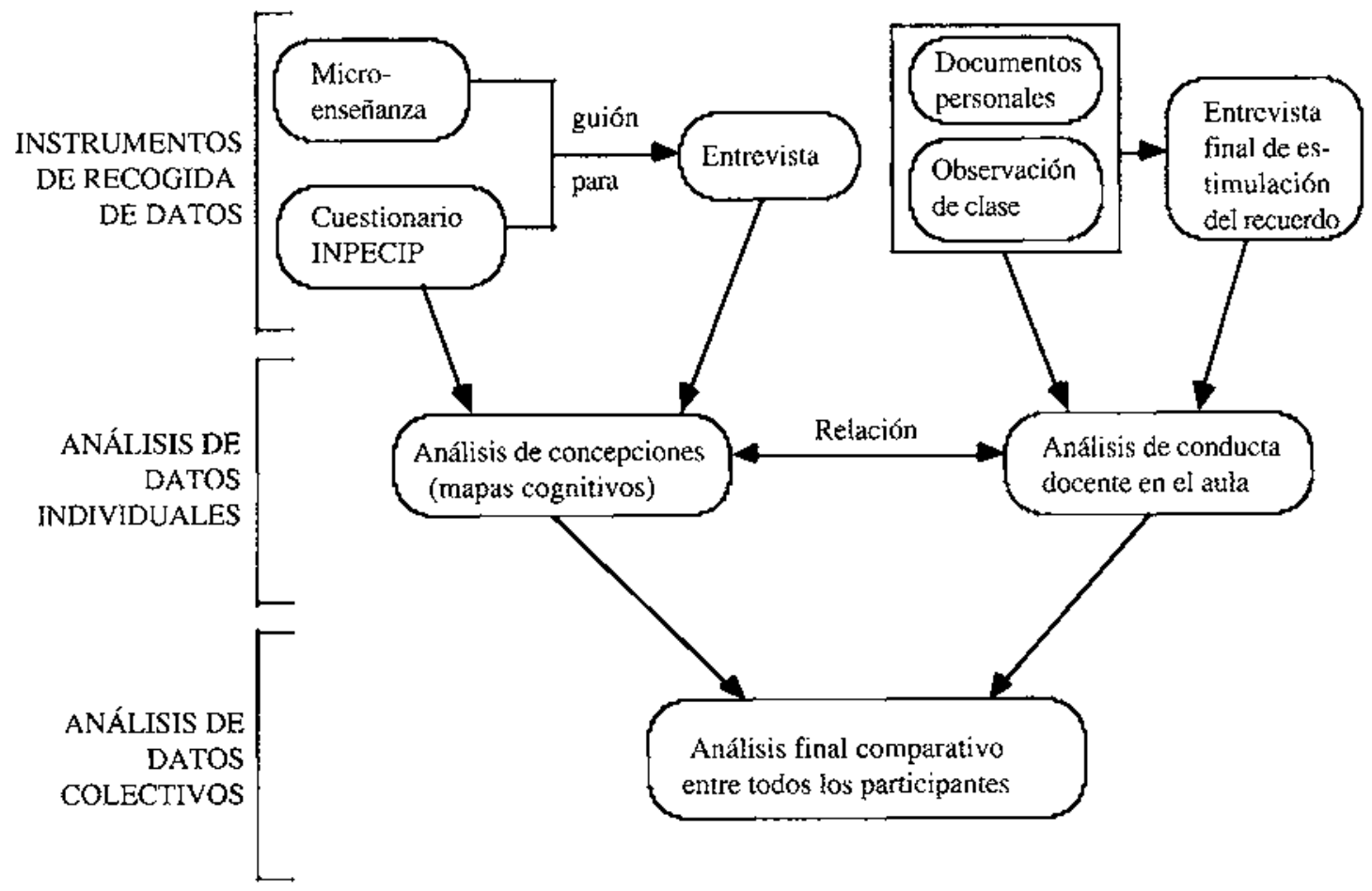

alguna manera que el alumno lo enticnda] ${ }^{7}$. Entonces [explicas y, si no comprenden, pues vuclta atrăs y a cxplicarto de otra forma ] $^{\mathrm{R}}$. (Aunque les tengas que dar un enunciado, porque hay veces que hay que considerar el lenguaje que maneja la física, y que hay que darle las cosas con ese lenguaje para que se familiaricen, sobre todo sì van a hacer ciencias] ${ }^{9}$. Pcro tambićn [conviene explicarlo a tu manera, decirlo con las palabras que el alumno maneja] ${ }^{10}$. Está bien que le digas que un cuerpo, cuando se ejerce una fuerza sobre él, adquiere una aceleración proporcional, pero también que se lo expliques a su manera. [La explicación no sería tanto dar un rollo, sino los dos o tres conceptos que hay que tener y tratar de darle la vuelta con sus palabras]'".

En la figura 2 se muestra el mapa cognitivo de David sobre la explicación, elaborado a partir de sus respuestas en la entrevista inicial. La numeración corresponde a la codificación de las preguntas de la entrevista. El mapa cognitivo mostrado es uno de $\operatorname{los} 32$ que hemos elaborado sobre las concepciones previas de David (Mellado, $1995 \mathrm{a})$.

\section{RESULTADOS}

\section{Concepciones previas de los profesores de ciencias en formación de primaria y secundaria}

En los cuatro profesores en formación estudiados se detecta una falta de reflexión previa sobre la naturaleza del conocimiento científico. No tienen una concepción definida sobre el conocimiento científico coherente en todos sus aspectos $y$, más que de una concepción única para cada profesor, deberíamos hablar de orientaciones o tendencias dominantes, pero manteniendo contradiciones. Estas contradicciones son fruto de la falta de reflexión sobre estos problemas y porque tienen profundamente asumidas una serie de ideas tópicas sobre «el método científico».

David considera que el conocimiento científico sólo refleja nuestro conocimiento de la realidad, no ta realidad misma. Para David, las tcorías cambian cuando se encuentra un experimento crucial que falla, es decir, las teorías no se prueban por verificación sino por falsación, tal como defendía Popper (1983); tiene también rasgos de la metodología de Lakatos (1981) cuando defiende que las teorías se reformulan por confrontación entre sí; $\mathrm{y}$, por último, piensa que las teorías cambian por otras que resuelven más problemas, tal como indicaba Laudan (1986). Por otra parte, David considera básica la prueba experimental, y se quedaría con las teorías que resuelvan mús problemas y que sean más sencillas, más elegantes y más globales; para ćl lo ideal sería una teoría que lo explicase todo. Sin embargo, cuando se refiere a la metodología científica, David tiene fuertes contradicciones entre el método científico empirista verificacionista y otras mctodologías en las que las ideas y teorías previas condicionan la observación. En general se muestra mucho más partidario del método científico empiris- 
Figura 2

Mapa cognitivo de David sobre la explicación del profesor, elaborado a partir de la entrevista inicial.

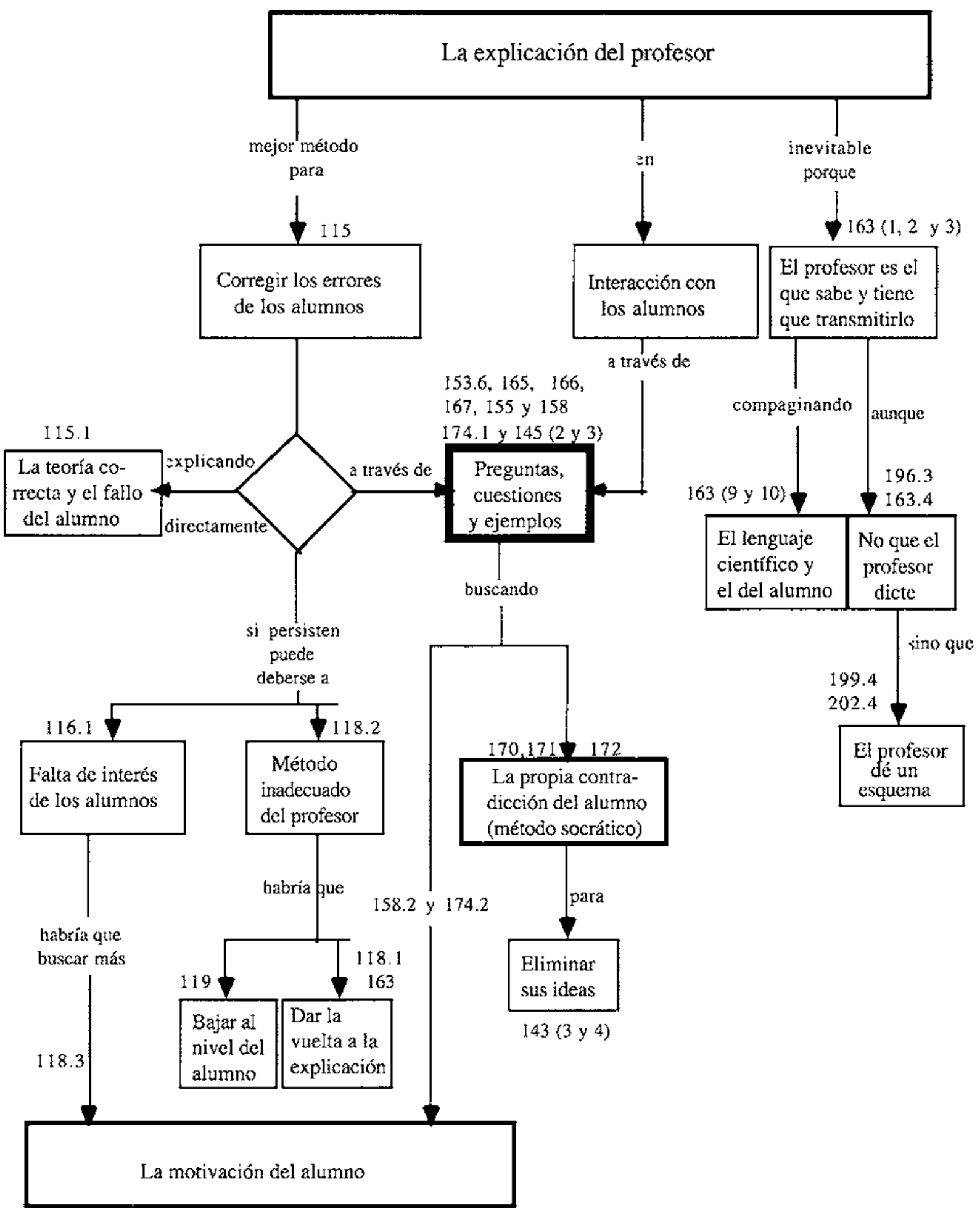


ta, que comienza con la observación y en el que la experimentación cumple el papel de comprobación (O-H-E-T: Observación-Hipótesis-ExperimentaciónTeorías).

Para Miguel el conocimiento científico tiene la misma categoría que otros conocimientos. Defiende una postura relativista de la ciencia próxima a la de Feyerabend (1987), ya que piensa que la ciencia depende de cada cultura y no hay criterios universales de demarcación. Los nuevos problemas y necesidades son los que hacen que avance el conocimiento científico. Migueles el más crítico con el método cientŕfico empirista. Piensa que se parte de la existencia de problemas a los que hay que dar soluciones y que no conviene encasillarse en un método rígido.

Ana es la que se muestra más positivista. Para ella las teorías se prueban por verificación experimental de hipótesis, formuladas a partir de observaciones previas. Ana cree que existen criterios universales y objetivos, aunque a veces influyen factores extracientíficos. Los criterios vendrían definidos por la aplicación del método científico empirista, a través del que se llega a teorias que son un reflejo cierto de la realidad. Aunque Ana también piensa que la metodología no tiene por qué ser única, su orientacion está fuertemente marcada por el método científico empirista como conjunto de pasos que comienza con la observación, seguida de la formulación de hipótesis, y la experimentación, que sería imprescindible para comprobar las hipótesis (O-H-E-T).

Julto piensa que el conocimiento científico es superior al resto de conocimientos. En el cambio de teorías científicas intervienen varios factores, desde nuevos descubrimientos a factores extracientíficos. Este profesor se quedaría con la teoría de que se cumpla en más situaciones diferentes. En cuanto a la metodología científica, también en Julio hay dudas y contradicciones, aunque aboga más por el método científico empirista que comienza con la observación.

Como Lederman (1992), no encontramos una relación significativa entre los antecedentes escolares de los profesores y sus concepciones sobre la naturaleza de la ciencia: los licenciados tienen más conocimientos de ciencias, pero igual que los maestros muestran contradicciones y falta de reflexión previa. Los resultados de nuestra investigación muestran un panorama más complejo que las investigaciones precedentes que asignaban a los profesores de ciencias concepciones empíricopositivistas sobre la naturaleza de la ciencia. También en Jos valores éticos de la ciencia se observan diferencias con otros trabajos (Luffiego et al., 1994): los cuatro profesores creen que la ciencia será buena o mala dependiendo del uso que se haga de ella, y no comparten la creencia positivista de que el desarrollo de la ciencia y la técnica permitirá resolver los problemas de la humanidad y conducirá al progreso social.

Coincidimos plenamente con las investigaciones que señalan que la filosofía de la ciencia apenas es tratada en los programas de formación de profesores de ciencias ni en las carreras de ciencias, y que debería abordarse para ayudar a los profesores a reflexionar y clarificar sus propias concepciones epistemológicas (Acevedo, 1994; Aguirre et al., 1990; Anderson, 1989; Cachapuz, 1994; Pomeroy, 1993; Smith y Neale, 1991). Los profesores de ciencias tienden a infravalorar las cuestiones de filosofía de la ciencia porque no han reflexionado antes sobre ellas (Lakin y Wellington, 1994).

En cuanto a sus concepciones sobre el aprendizaje de las ciencias, los cuatro profesores reflejan una aparente orientación constructivista del aprendizaje, como construcción activa a partir de las ideas previas de los alumnos. Sin embargo, el valor epistemológico que dan a las ideas de los alumnos es muy diferente para cada caso. David y Julio consideran importante conocer las ideas de los alumnos, pero no les dan valor epistemológico, sino que las consideran simples errores que el profesor tiene que eliminar si no coinciden con las de la ciencia. Miguel, en cambio, considera las ideas de los alumnos verdaderas teorías alternativas, con el mismo valor epistemológico que las del currículo escolar. En consecuencia, el profesor no tiene que cambiarlas, sino ayudar a los alumnos a que las refuercen y justifiquen por sí mismos. Esta concepción es coherente con su orientación relativista sobre el conocimiento científico. Para Ana, el valor epistemológico lo tiene el currículo escolar y no las ideas de los alumnos. Sin embargo, esta maestra da mucha importancia a las ideas de los niños, por el hecho de que han sido elaboradas por los niños, que para ella son los protagonistas de la clase. Aunque considere que las ideas intuitivas son erróneas, no trataría de rebatirlas, ya que para ella la adquisición de conocimientos esta supeditada a la formación integral de los niños.

En relación con las concepciones sobre la enseñanta de las ciencias, en todos los profesores en formación estudiados coexisten rasgos de varios modelos, a veces de forma contradictoria. También aquí nos referiremos a orientaciones o tendencias dominantes para cada uno, sin excluir que existan contradicciones en algunos aspectos.

Según el cuestionario, los cuatro profesores planificarían por objetivos conductuales; sin embargo, en la entrevista rechazan la planificación por objetivos y defienden una planificación por contenidos. Los maestros además planificarían actividades. La discrepancia entre las contestaciones en el cuestionario y en la entrevista, las hemos detectado en varios aspectos y coincidimos con Lederman y O'Malley (1990) y Gunstone y otros (1993) en la limitación del cuestionario para detectar las concepciones de los profesores.

Los cuatro profesores consideran muy importante la motivación para la enseñanza de las ciencias. Creen que hay que motivar a los alumnos a través de curiosidades, anécdotas, problemas de actualidad, cuestiones que tengan relación con la vida cotidiana, etc.

En cuanto a las estrategias de enseñanza, David tendría como eje de la enseñanza la explicación del profesor, aunque también consideraría una estrategia de cambio 
conceptual simple, basado en la contradicción entre las ideas de los alumnos y las del currículo escolar. AI cambio conceptual llegarían los alumnos a través del diálogo o de la explicación del profesor. Miguel cree que Ios alumnos deben debatir sus ideas en clase para reforzar y justificar sus pensamientos, y la explicación de! profesor no tiene la misión de rebatir, sino de aportar un elemento más al debate. Ana defiende una estrategja de cambio conceptual simple, guiada y orientada por la maestra a través del diálogo, de actividades y de la propia explicación. Julio coincide con David en que hay que eliminar las ideas erróneas de los niños a través de la contradicción o de la explicación del profesor.

Las concepciones de los profesores en formación sobre la enseñanza de las ciencias están muy relacionadas con sus concepciones sobre el aprendizaje de las ciencias. En cambio, los resultados de nuestra investigación nos indican que no es posible establecer un isomorfismo entre las concepciones de los profesores sobre la ciencia y sobre enseñanza y aprendizaje de las ciencias. La concepción de Miguel sobre la enseñanza y aprendizaje de las ciencias es coherente con su concepción relativista sobre la naturaleza de la ciencia. En cambio, Ana tiene una orientación parcialmente constructivista sobre la enseñanza de las ciencias, mientras que su concepción sobre la naturaleza de las ciencias está más orientada al positivismo empirista. I os otros dos profesores estudiados (David y Julio) tienen una correspondencia parcial entre sus concepciones sobre la ciencia y sus concepciones sobre la enseñanza y el aprendizaje de las ciencias.

\section{Conducta docente durante la impartición de la lección «Energía y medio ambiente»}

I os dos licenciados y el maestro planifican por contenidos. Sólo la maestra hace una planificación más completa que incluye objetivos, contenidos, actividades, metodología, evaluación, etc. Sin embargo los cuatro tienen una finalidad u objetivo personal, implícito, que condiciona toda su actuación (Brickhouse, 1993; Duschl y Wright, 1989). Este objetivo general no es señalado en la planificación sino en la entrevista final de estimulación del recuerdo.

David y los dos maestros (Ana y Julio) dan al tema una orientación de «ciencia, tecnología y sociedad». Miguel se centra en Ia relación de la energía y los seres vivos. El contenido tiene una estructura lógica y ordenada en los dos licenciados y en Ana. En Julio el contenido es desordenado, anecdótico y sin una estructura lógica interna. El contenido tiene una buena estructura psicológica para David y Ana. En cambio, en Miguel, excepto en el concepto de energía, prima la estructura lógica sobre la psicológica y los contenidos son demasiado extensos para desarrollar en la clase. David tiene como principio físico integrador el de conservación de la energía, Miguel, el de degradación de la energía y los dos maestros, el de transformación de la energía. La orientación más abierta de la ciencia reflejada en el aula corresponde a David. La visión más cerrada del conocimiento científico la ofrece Miguel, que se limita a transmitir un cuerpo de conocimientos ya elaborados; esta orientación de Miguel se contradice fuertemente con su concepcion previa relativista de la ciencia.

El tratamiento didáctico del concepto de energía es descriptivo en Miguel, comenzando por el conocimiento de las distintas energías, y a partir de la definición de trabajo mecánico en los otros tres. Tanto desde el punto de vista científico (Feynman, 1971) como del didáctico (Hernández, 1993; Varela et al., 1993) parece que es más adecuado comenzar el tema de la energía de una forma descriptiva. David y los dos macstros dan una gran importancia al desarrollo de actitudes en los alumnos. En cambio, Miguel apenas genera actitudes en los estudiantes.

En el tema «Energía y medio ambiente» no puede establecerse una estructura típica del contenido que diferencie a los licenciados de los maestros.

En su concepción previa, los cuatro profesores manifestaron que partirían de las ideas intuitivas de los alumnos, almque el significado de dichas ideas era diferente para cada uno. En el aula ninguno de los profesores hace un diagnóstico sistemático e individualizado de las ideas de los niños, lo que dificulta que realmente puedan partir de ellas y hacer un seguimiento individualizado del aprendizaje. Piensan más en términos globales sobre el grupo de clase que diferenciadamente sobre los individuos (Neale et al., 1987).

David comienza preguntando a los alumnos más como una estrategia de motivación y de participación que de cambio conceptual. Sin embargo, concede valor didáctico a las ideas de los alumnos, que tiende a reforzar y a guiar a través de nuevas preguntas y no a rebatir. En cuanto a las estrategias didácticas, la concepción previa de David estaba entre un modelo transmisivo y uno de cambio conceptual simple que se produciría por la contradicción del propio alumno o por la explicación del profesor. En el aula, David utiliza el diálogo y sobre todo la explicación del profesor, en una estrategia básicamente transmisiva, aunque con participación de los estudiantes. En cambio, no utiliza estrategias de contradicción, y su conducta es sólo parcialmente coherente con su concepción previa.

En el aula, Miguel considera a los alumnos meros receptores pasivos de conocimiento externo, en contra de su concepción previa del aprendizaje de las ciencias. $\mathrm{Mi}$ guel sigue una estrategia de transmisión de conocimiento externo, basada en la explicación del profesor. Su ritmo es muy rápido y sin pausas, lo que dificuita la participación y la asimilación de los estudiantes (Tobin et al., 1994). Para él lo más importante es completar los contenidos previstos. Esta estrategia es completamente opuesta a su concepción previa, que pretendía reforzar las ideas de los estudiantes a través del debate.

En la clase, Ana da valor didáctico a las ideas de los alumnos, aunque no valor epistemológico. Comienza preguntando a los estudiantes $y$, a partir de sus ideas, 
plantea nuevas preguntas-guías que refuercen las ideas de los niños. Sus explicaciones son cortas, de aclaración y refuerzo y para relacionar las nuevas ideas con las de los niños. Ana sigue en el aula una estrategia de cambio conceptual guiado a través del diálogo, de actividades y de la propia explicación. Los alumnos de Ana son los únicos que están colocados en mesas de seis y realizan actividades en grupo. La conducta docente de Ana es bastante coherente con su concepción previa del aprendizaje y de la enseñanza de las ciencias.

Julio tiene una concepción previa situada entre la transmisión verbal de conocimientos y el cambio conceptual simple a través de la contradicción y, sobre todo, de la explicación del profesor. En el aula no utiliza la contradicción y pregunta a los alumnos más como una estrategia de motivación y de participación que de cambio conceptual. Sus preguntas son de bajo nivel cognitivo y apenas tienen seguimiento. Su conducta es s6lo parcialmente compatible con su concepción previa.
Para los profesores en formación estudiados, los resultados de nuestra investigación no permiten establecer una correspondencia entre las concepciones de los profesores sobre la naturaleza de la ciencia y la conducta en cI aula. La profesora en formación con una concepción más positivista sobre la ciencia (Ana) es la más constructivista en el aula. En cambio, el profesor en formación con una concepción relativista sobre la ciencia (Miguel) aplica en el aula un modelo didáctico tradicional transmisivo. Coincidimos con los trabajos de Lederman y colaboradores, que no encuentran de forma general una relación entre las concepciones epistemológicas de los profesores y la práctica del aula. Al menos en los profesores de ciencias en formación no es automática Ia transferencia entre las concepciones previas sobre la naturaleza de la ciencia y la práctica del aula.

En cuanto a la correspondencia entre las concepciones de los profesores sobre la enseñanza y aprendizaje de las ciencias y la práctica del aula, existe bastante coherencia

Figura 3

Relación entre las concepciones previas y la conducta en el aula.

\begin{tabular}{|c|c|c|c|c|c|}
\hline & & David & Miguel & Ana & Iulio \\
\hline \multirow{2}{*}{ 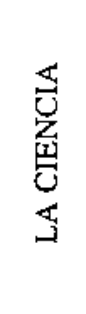 } & $\begin{array}{c}\text { Concepción } \\
\text { previa }\end{array}$ & $\begin{array}{l}\text { Entre el positivis- } \\
\text { mo empirista y } \\
\text { la construcción } \\
\quad \text { social }\end{array}$ & Relativismo & $\begin{array}{l}\text { Positivismo } \\
\text { empirista }\end{array}$ & $\begin{array}{l}\text { Entre el positivis- } \\
\text { mo empirista y la } \\
\text { construcción social }\end{array}$ \\
\hline & $\begin{array}{l}\text { Conducta } \\
\text { en el aula }\end{array}$ & $\begin{array}{l}\text { La ciencia como } \\
\text { construcción social }\end{array}$ & Positivismo & $\begin{array}{l}\text { Entre el positivismo } \\
\text { y la ciencia para el } \\
\text { desarrollo humano }\end{array}$ & $\begin{array}{l}\text { Entre el positivis- } \\
\text { mo empirista y la } \\
\text { construcción social }\end{array}$ \\
\hline \multirow{2}{*}{ 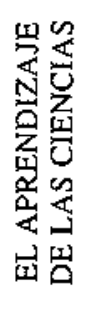 } & $\begin{array}{l}\text { Concepción } \\
\text { previa }\end{array}$ & $\begin{array}{l}\text { Constructivismo } \\
\text { simplificado }\end{array}$ & $\begin{array}{l}\text { Constructivismo } \\
\text { complejo y } \\
\text { relativista }\end{array}$ & $\begin{array}{l}\text { Constructivismo } \\
\text { simplificado y } \\
\text { humanista }\end{array}$ & $\begin{array}{l}\text { Constructivismo } \\
\text { simplificado }\end{array}$ \\
\hline & $\begin{array}{l}\text { Conducta } \\
\text { en el aula }\end{array}$ & $\begin{array}{l}\text { Pregunta las ideas } \\
\text { de los alumnos } \\
\text { como motivacion } \\
\text { y participacion }\end{array}$ & $\begin{array}{l}\text { El alumno como } \\
\text { receptor pasivo } \\
\text { de conocimiento } \\
\text { externo }\end{array}$ & $\begin{array}{l}\text { Constructivismo } \\
\text { simplificado y } \\
\text { humanista }\end{array}$ & $\begin{array}{l}\text { Pregunta las ideas } \\
\text { de los alumnos } \\
\text { como motivación } \\
\text { y participación }\end{array}$ \\
\hline \multirow{2}{*}{ 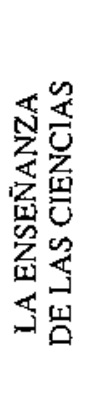 } & $\begin{array}{c}\text { Concepción } \\
\text { previa }\end{array}$ & $\begin{array}{l}\text { Entre la transmisión } \\
\text { por explicación } \\
\text { y el cambio } \\
\text { conceptual por } \\
\text { contradicción }\end{array}$ & $\begin{array}{l}\text { Relativismo } \\
\text { pedagógico } \\
\text { (debate para } \\
\text { reforzar las ideas } \\
\text { de los alumnos) }\end{array}$ & $\begin{array}{l}\text { Cambio conceptual } \\
\text { guiado a través } \\
\text { del diálogo, de } \\
\text { actividades o de } \\
\text { la explicaciôn }\end{array}$ & $\begin{array}{l}\text { Entre la transmisión } \\
\text { por explicación } \\
\text { y el cambio } \\
\text { conceptual por } \\
\text { contradicción }\end{array}$ \\
\hline & $\begin{array}{l}\text { Conducta } \\
\text { en el aula }\end{array}$ & $\begin{array}{l}\text { La explicación eje } \\
\text { de la clase con } \\
\text { participación de } \\
\text { los alumnos }\end{array}$ & $\begin{array}{l}\text { Transmisión tra- } \\
\text { dicional de cono- } \\
\text { cimiento externo, } \\
\text { sin participación } \\
\text { de los alumnos }\end{array}$ & $\begin{array}{l}\text { Cambio conceptual } \\
\text { guiado a través } \\
\text { del dialogo, de } \\
\text { actividades o de } \\
\text { la explicacion }\end{array}$ & $\begin{array}{l}\text { La explicación eje } \\
\text { de la clase con } \\
\text { participación de } \\
\text { los alumnos }\end{array}$ \\
\hline
\end{tabular}


en Ana y una correspondencia parcial en David y Julio. En cambio, hay una fuerte contradicción en Miguel. Tampoco en este aspecto, al menos para los profesores en formación estudiados, podemos establecer de una forma nítida la relación entre las concepciones de los profesores sobre la enseñanza y el aprendizaje de las ciencias y la práctica del aula. Hay que considerar otros factores que pueden influir de una forma determinante. En la figura 3 resumimos estas conclusiones.

Finalizamos este apartado señalando algunas de las limitaciones que hemos detectado a lo largo de nuestra investigación y de las que queremos dejar constancia para futuras investigaciones. En primer lugar, al no realizarse una rigurosa selección por criterios de los participantes, no podemos garantizar que sean suficientemente representativos de sus colectivos. En segundo lugar, hay aspectos de la conducta docente en el aula, como la evaluación del aprendizaje de los alumnos, que no se han observado en las grabaciones de clase realizadas. En tercer lugar, con el estudio realizado hemos obtenido una visión en un momento dado de las concepciones y conducta docente de los profesores; sin embargo, creemos que la propia participación en la investigación ha supuesto un instrumento de intervención didáctica para los profesores participantes, por lo que un estudio longitudinal posterior nos hubiera permitido seguir la evolución de las concepciones y conducta en el aula de los profesores participantes.

\section{IMPLICACIONES PARA LA FORMACIÓN DEL PROFESORADO DE CIENCIAS}

Cuando el profesor de ciencias en formación comienza sus estudios universitarios, Io hace con unos conocimientos, valores, creencias y actitudes sobre la ciencia, la enseñanza y aprendizaje de las ciencias, el profesor, etc., fruto de sus anos previos de escolaridad. Durante su etapa de formación inicial, el profesor de ciencias tiene que aprender una serie de conocimientos profesionales en dos aspectos diferenciados, aunque estrechamente relacionados entre sí, y que denominamos componentes estática y dinámica (Blanco et al., 1995; Mellado, 1995b).

En ta componente estática incluimos aquellos conocimientos académicos que pueden ser independientes de la persona concreta que enseña y del contexto específico donde se desarrolla la actividad docente. Esta parte puede ser adquirida en materiales escritos o andiovisuales sin implicación personal directa. En esta componente incluimos, entre otros, los conocimientos de ciencias, los conocimientos psicopedagógicos generales y los conocimientos térícos de didáctica de las ciencias; los dos últimos, casi ausentes en la formación inicial del profesorado de secundaria. El conocimiento proposicional, académico o estático es necesario para el profesor de ciencias, pero no es suficiente para que el profesor en formación aprenda a enseñar ciencias.

Además, los profesores en formación tienen que reflexionar sobre sus concepciones sobre la ciencia y sobre el aprendizaje y enseñanza de las ciencias; pero la reflexión sobre sus concepciones no garantiza de forma automática su transferencia a la práctica del aula.

En nuestra opinión, existe una componente profesional de los profesores que denominamos «dinámica» (Fig. 4), y que tiene un estatus diferente que la componente estática (Blanco et al., 1995, Mellado, 1995b; Pro, 1995). La componente dinámica se genera y evoluciona a partir de los propios conocimientos, creencias y actitudes, pero requiere de la implicación y reflexión personal y de la práctica de la enseñanza de la materia específica en contextos escolares concretos. Este proceso permite al profesor reconsiderar su conocimiento estático y sus concepciones, modificándolos o reafirmándolos. Es también, como señalan Wilson y otros (1987), una forma de razonamiento y acción pedagógica en la enseñanza de la materia específica.

La componente dinámica es la más específicamente profesional y la que distingue a los profesores de ciencias expertos de los principiantes. A lo largo de sus años de enseñanza, el profesor experto va desarrollando la componente dinámica e integra en una estructura única las diferentes componentes del conocimiento. Coincidimos con Gess-Newsome y Lederman (1993), Hauslein y otros (1992) y Lederman y otros (1994) cuando consideran que esta estructura única es el conocimiento didáctico del contenido (CDC).

Durante las prácticas de enseñanza, los estudiantes para profesores pueden ir generando sus propios esquemas prácticos de acción en la enseñanza de las ciencias. La reflexión en y sobre la práctica de la enseñanza (Schön, 1983) permite al profesor en formación analizar su conducta en clase y contrastarla con sus concepciones previas (Louden y Wallace, 1994) en un proceso de retroacción continuo (Villar, 1990). También le permite, a través del estudio de casos en el centro universitario, contrastar su conducta docente con las de profesores expertos de ciencias y con las de sus propios compañeros. Para ello tienen que reflexionar con sus supervisores universitarios, profesores-tutores y compañeros, redefinir sus estrategias de enseñanza, contrastarlas con sus creencias previas y volverlas a poner en práctica. Hacker (1988) señala que al menos se necesitan tres períodos de prácticas para que el profesor de ciencias en formación inicial aumente sus conductas intelectuales profesionales. En este proceso es fundamental el apoyo que el estudiante que estudia para profesor reciba del supervisor universitario, del tutor del centro de prácticas y de sus propios compañeros, ya que su aprendizaje incluye el desarrollo social y personal junto al desarrollo profesional (Bell y Gilbert, 1994). Las creencias del profesor en formación serán consistentes con su práctica y ambas podrán cambiar en la medida que contemple estos tres aspectos. Las creencias del profesor pueden influir como una variable más en la implementación del currículo, pero también éste influye en las creencias del profesor (Tobin et al., 1994).

Otro aspecto a destacar es la metodología utilizada en la formación de profesores. Si los profesores en formación 
Figura 4

Componentes del conocimiento profesional del profesor de ciencias.

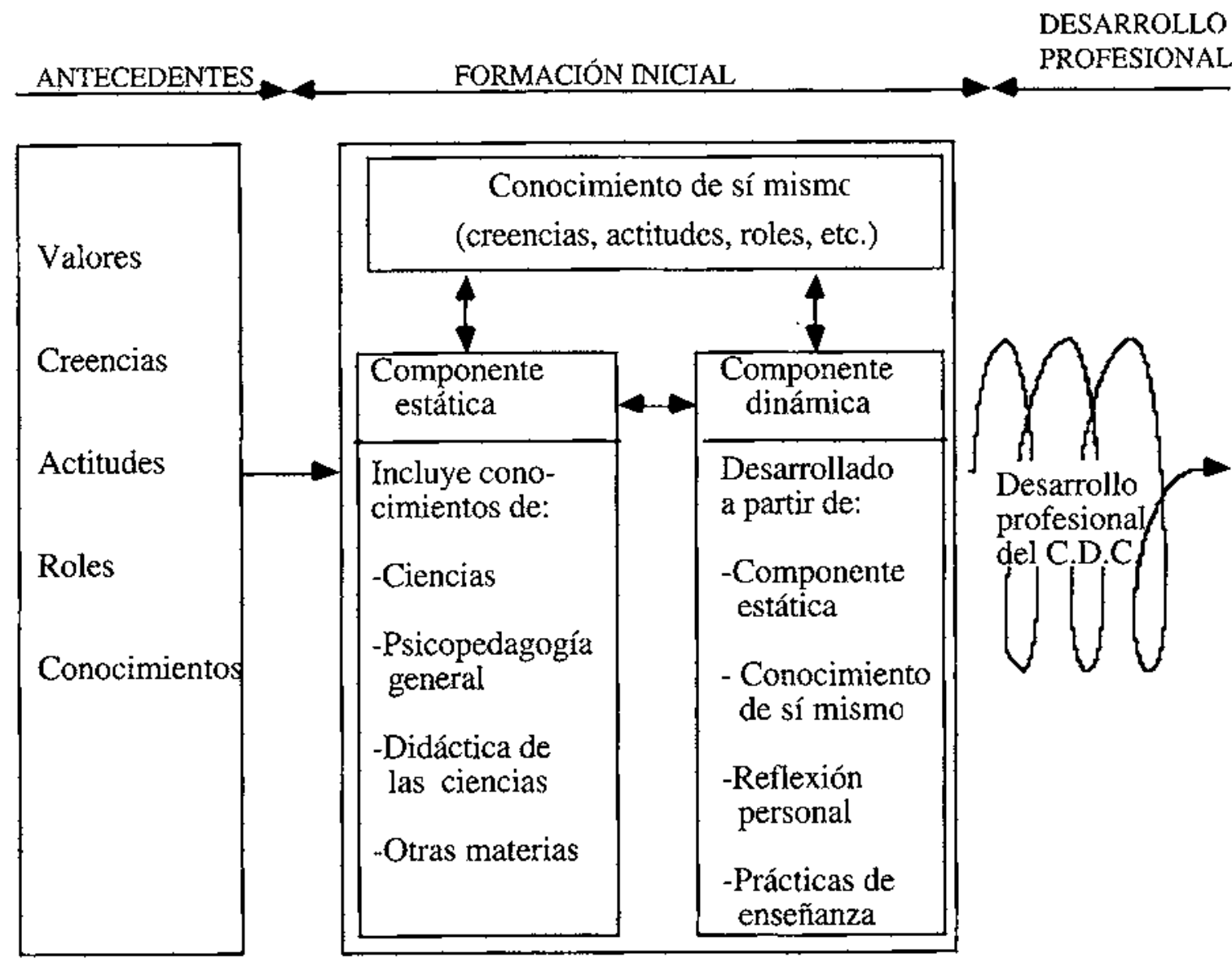

toman como referencia, positiva o negativa, para la enseñanza de las ciencias, a los profesores que han tenido a lo largo de su etapa escolar, es fundamental que la metodología utitizada durante la formación inicial por los formadores de profesores sea consistente con los modelos teóricos que propugnan. En caso contrario, los estudiantes para profesores aprenderán más de lo que ven hacer en clase, que de lo que se les dice que hay que hacer (Fernández, 1994; Stoddart et al., 1993; Tobin et al. 1994).

Finalizamos destacando que la formación inicial de profesores de ciencias no puede limitarse al conocimiento proposicional estático, sino que tiene que introducir más conocimiento procedimental y esquemas estratégicos de acción -componente dinámica-para que el profesor en formación pueda asimilario como algo perso. nal, en un contexto de enseñanza práctica, y a partir de la reflexión de sus conocimientos científicos, de sus propias concepciones y de su propia práctica de enseñanza. (Blanco, 1994; Kagan, 1992). En este enfoque, las asignaturas de didáctica de las ciencias deben jugar un importante papel (Furió et al., 1992), especialmentc para el profesorado de secundaria. Otro aspecto esencial, y no resuelto todavía en España, sería el modelo organizativo universitario más adecuado para la formación inicial del profesorado de ciencias de educación secundaria obligatoria.

\section{REFERENCIAS BIBLIOGRÁFICAS}

ABIIL, S.K. y SMITH, D.C. (1994). What is science?: preservice elementary teachers' conceptions of the nature of science. International Journal of Science Education, 16(4), pp. 475-487.
ACEVEDO, J.A. (1994). Los futuros profesores de enseñanza secundaria ante la sociología y la epistemología de las ciencias. Un enfoque C-T-S. Revista Interuniversitaria de Formación del Profesorado, 19, pp. 111-125. 
AGUIRRE, M. y HAGGERTY, S. (1995). Preservice teachers" meanings of learning, International Journal of Science Education, 17(1), pp. 119-131.

AGUIRRE, M., HAGGERTY, S. y LINDER, C. (1990). Siudentteachers' conceptions of science teaching and learning: a case study in preservice science education. International Journal of Science Education, 12(4), pp. 381-390.

ANDERSON, C.W. (1989). Policy implications of research on science teaching and teachers' knowledge, en Competing Vision of Teacher Knowledge, East Lansing National Center for Research on Teacher Education, pp. 1-28.

ANDERSON, R.D. y MITCHENER. C.P. (1994). Research in Science Teacher Education, en Gabel, D.L. (ed.), Handbook of Research on Science Teaching and Learning, po. 3-44. Nueva York: Mcmillan.

BALLLNILLA, F. (1992). El cambio de modelo didáctico, un proceso complejo. Investigación en la Escuela, 18, pp. $43-68$.

BELL, B. y GILBERT, J. (1994). Teacher Development as Professional, Personal and Social Development. Teaching \& Teacher Education, 10(5), pp. 483-497.

BENSON, G. (1989). Epistemology and science curriculum. Joumal of Curriculum Studies, 21(4), pp. 329-344.

BLANCO, L. (1991). Conocimiento y acción en la enseñanza de las matemáticas de profesores de EGB y estudiantes para profesores. Badajoz: S. Publicaciones UEX.

BLANCO, L. (1994). Initial training and teaching practice. Methodological issues in learning to teach. First ltalianSpanish research symposium on mathematics. Universitá di Modena (Italia): Dpto. di Matemática.

BI.ANCO, L. MEI,LADO,V y RUIZ, C. (1995). Conocimiento didáctico del contenido en ciencias experimentales y matemáticas y formación del profesorado. Revista de Educación, 307, pp, 427-446.

BRICKHOUSE, N.W. (1990). Teachers' beliefs about the naturc of science and their relationship to classroom practice. Journal of Teacher Hiducation, 41(3), pp. 53-62.

BRICKHOUSE, N.W. (1993). What counts as successful instruction? An account of a teacher's self-assessment. Science Education, 77(3), pp. 115-129.

BRISCOE, C. (1993). Using cognitive referents in making sense of teaching: A chemistry teacher's struggle to change assessment practice. Journal of Research in Science Teaching, 30(8), pp. $971-987$.

CACHAPUZ, A. (1994). Filosofia da cience e ensino da quimica: Repensar o papel do trabalho experimental, en Montero, M.L. y Vez, J.M. (cds.), Las didácticas específicas en la formación del profesorado $1 /(l)$, pp. 357-364. Santiago: 'Tórculo.

CARLSHN, W.S. (1993). Teacher knowledge and discourse control: Quantitative evidence from novice biology teachers' classrooms. Journal of Research in Science Teaching, 30(5), pp. 471-481.

CRONIN-JONES, L.L. (1991). Science teaching beliefs and their influence on curriculum implementation: Two case studies. Journal of Research in Science Reaching, 38(3), pp. $235-250$.

CURRAIS, J. y PÉRE7, M. (1994). Epistemología y enseñanza de las ciencias: implicaciones en la formación del profesorado, en Montero, M.I. y Vez, J.M. (eds.), Las didácticas espe- cificas en la formación del profesorado $I(I)$, pp. 419-424. Santiago: Tórculo

DILI.ON, D.R., O'BRIEN, D.G., MOJE, E.B. y STHWART, R.A. (1994). Literacy learning in secondary school science classrooms: Across-case analysis of three qualitative studies. Journal of Research in Science Tcaching, 31(4), pp. 345362.

DUSCHL, R.A. y WRIGHT, E. (1989). A case study of high school teachers' decision making models for planning and teaching science. Journal of Research in Science Teaching, 26(6), pp. 467-501.

ESTEBARANZ, A. (1992). El análisis de datos cualitativos a través de tablas de contingencia, en Marcelo, C. (cd.), la investigación sobre la formactón del profesorado. Métodor de investigación y análisis de datos, pp. 147-170. Buenos Aires: Cincel.

FERNÁNDEZ, M. (1994). Las tareus de la profesion de anseñar. Madrid: Siglo XXI.

FEYFRABEND, P. (1987). Contra elmétodo. Barcelona: Ariel.

FEYNMAN, R. et al. (1971). The Feynman. lectures on Physics. F.Ed. Iberoamericano. EEUU.

FREIRF, A.M. y CHORÄO, M.Ki. (1992). Elements for a typology of teachers' conceptions of physics teaching. Teaching and Teacher Education, 8(5-6), pp. 497-507.

FURIó, C. (1994). Tendencias actuales en la formación del profesorado de ciencias. Enseñanza de las Ciencias, 12(2), pp. 188-199.

FURIÓ, C., GIL, D., PESSOA DF CARVALHO, A.M. y SALCEDO, L.E. (1992). La formación inicial del profesorado deeducacion secundaria: papel de las didácticas especiales. Investigación en la Escuela, 16, pp. 7.21.

GALLAGHER, J.J. (1991). Prospective and practicing secondary school science teachers' knowledge and beliefs about the philosophy of science. Science Education, 75(1), pp. 12I133.

GESS-NEWSOME, J, y LEDERMAN, N.G. (I993). Preservice biology teachers' knowledge structures as a funtion of professional teacher education: A year-long assessment. Science Education, 77(1), pp. 25-45.

GrL, D. (1993). Contribución de la historia y de la filosofía de tas ciencias al desarrollo de un modelo de enseñanzaaprendizaje. Enseñanza de las Ciencias, 1 I(2), pp. 197-2!2.

GOETZ, J.P. y LECOMPTE, M.D. (1988). Ethografia y diseño cualitativo en investigación cualitativa. Madrid: Morata.

GON'LÁlLEZ, F.M. (1992). Los mapas conceptuales de J.D. Novak como instrumento para la investigación en didáctica de las ciencias experimentales. Enseñanza de las Ciencias, 10(2), pp. $148-158$.

GUNSTONE, R.F., SLATTERX, M, BAIR, J.R. y NORTHFIELD, J.R. (1993). A case study exploration of development in preservice science teachers. Science Education, $77(1)$, pp. 47-73.

HACKER, R.G.(1988). A model for the professional development of student teachers of science. International Journal of Science Education, 10(2), pp. 203-210.

HAUSLEIN, P.L., GOOD, R.G. y CUMMINS, C.L. (1992). Biology content cognitive structure: From science student to science teacher. Journal of Research in Science Teaching, 29(9), pp. 939-964. 
HERNÁNDEZ, L.M. (1993). Tareas de planiftcación del módulo «I a energía y los recursos energéticos» en el marco de la formación del profesorado. Enseñanza de las Ciencias, I 1(3), pp. 247-254.

HEWSON, P.W. (1993). Constructivism and reflective practice in science teacher education, en Montero, M.I. y Vez, J.M., las didácticas especificas en la formación del profesorado, pp. 259-275. Santiago: Tórculo.

HEWSON, P.W. y HEWSON, M. G. (1989). Analysis and use of a task for identifyng conceptions of teaching science. Journal of Education for Teaching, IS(3), pp. 191-209.

HUIBREGTSE, I., KORTHAGEN, F. y WUBBELS, T. (1994). Physics teachers' conceptions of learning, teaching and professional development. International Joumal of Science Edacation, 16(5), pp. 539-561.

JIMÉNEZ, M.P. (1995). Comparando teorías: la reflexión epistemológica sobre la naturaleza de lacienciaen la formación del profesorado, en Blanco, L. y Mellado, V. (eds.), La formación del profesorado de ciencias y matemáticas en España y Portugal, pp. 267-280. Badajoz: Diputación Provincial.

KAGAN, D. (1992). Professional growth among preservice and beginning teachers. Review of Educational Research, $62(2)$, pp. $129-170$.

KOBALLA, T.R, y CRAWLEY, F.E. (1985). The influence of attitude on science teaching and learning, School Science and Mathematics, 85 , pp. 222-232.

KOULAIDIS, V. y OGBORN, J. (1989). Philosophy of science: an empirical study of teachers" views. International Journal of Science Education, 1I(2), pp. 173-184.

KOULAIDIS, V. y OGBORN, J. (1995). Science Teachers' Philosophical Assumptions: How well do we Understand them? International Journal of Science Education, 17(3), pp. 273-283.

KUHN, T. (1971). La estructura de las revoluciones cientificas. Madrid: Fondo de Cultura Económica.

LAKATOS, I. (1981), Matemáticas, ciencia y epistemología. Madrid: Alianza Universidad.

LAKIN, S. y WELLINGTON, J. (1994). Who will teach the «nature of science»?: Teachers' views of science and their implications for science edtcation. International Journal of Science education, 16(2), pp. 175-190.

LAUDAN, L. (1986). El progreso y sus problemas. Hacia una teoría del progreso cientifico. Madrid: Ediciones Encuentro.

LEDERMAN, N.G. (1986). Relating teaching behavior and classroom climate to changes in students' conceptions of the nature of science. Science Education, 70(1), pp. 3-19.

LEDERMAN, N.G. (1992). Students' and teachers' conceptions of the nature of science: A review of the research. Journal of Research in Science Teaching, 29(4), pp. 33I-359.

LEDERMAN, N.G., GESS-NEWSOME, J. y LATZ, M.S. (1994). The nature and development of preservice science teachers' conceptions of stubject matter and pedagogy. Journal of Research in Science Teaching, 31(2), pp. 129-146.

LEDHRMAN, N.G. y O'MALLEY, M. (1990) Students' perceptions of tentativeness in science: Development, use, and sources of change. Science Education, 74(2), pp. 225-239.
L.FDERMAN, N.G. y ZEIDLER,D.I. (1987). Science teachers" conceptions of the nature of science: Do they really influence teaching behavior? Science Education, 7 I (5), pp. 72 I-734.

LEE, O. y PORTER, A.C. (1993). A teacher's bounded rationality in middle school science. Teaching and Teacher Education, 9(4), pp. 397-409.

I_LINARES, S. (I992). Los mapas cognitivos como instrumento para investigar las creencias epistemológicas de los profesores, en Marcelo, C. (ed.), La investigación sobre la formación del profesorado. Métodos de investigación y análisis de datos, pp. 57-95. Argentina CF.: Cincel.

LÓPEZ, J.I. (1994). El pensamiento del profesor sobre el conocimiento de los alumnos. Investigación en la Escuela, $22, \mathrm{pp} .58-66$.

LÓPEZ RUPÉREZ, F. (1991). Organización del conocimiento y resolución de problemas de física. Madrid: CIIDF-MEC.

LORSBACH, A.W., TOBIN, K., BRISCOE, C. y LAMASTFR, S.U. (1992). An interpretation of assessment methods in middic school science. International Journal of Science Education, 14(3), pp. 305-317.

LOUDEN, W. y WALLACE, J. (1994). Knowing and Teaching Science: The Constructivist Paradox, International Journal of Science Education, 16(6), pp. 649-657.

LUFFIEGO, M. et al. (1994). Epistemología, caos y enseñanza de las ciencias. Enseñanza de las Ciencias, I2(2), pp. 89-96.

MARCELO, C. (1987). El pensamientodel profesor. Barcelona: CEAC.

MARCELO,C. (1992). Dar sentido a los datos: combinación de perspectivas cualitativa y cuantitativa en el análisis de las entrevistas, en Marcelo, C. (ed.), La investigación sobre la formación del profesorado. Métodos de investigación y análisis de datos, pp. 13-49. Argentina CF.: Cincel.

MARCELO, C. (1993). Cómoconocen los profesores la materia que enseñan. Algunas contribuciones de la investigación sobre conocimiento didáctico del contenido, en Montero, M.L. y Vez, J.M. (eds.), Las didacticas especificas en la formación del profesorado, pp. 151-186. Santiago: Tórculo.

MARCELO, C. (1994). Formación del profesorado para el cambio educativo. Barcelona: PPU.

MARCELO, C. (1995). Investigación sobre formación del profesorado: El conocimiento sobre aprender a enseñar, en Blanco, L. y Mellado, V. (eds.), La Formación del profesorado de ciencias y matemáticas en España y Portugal, pp. 3-35. Badajoz: Diputación Provincial.

MARCELO, C. y PARRILLA, A. (1991). El estudio de caso: Una estrategia para la formación del profesorado y la investigación didáctica, en Marcelo, C. et al. (eds.), El estudio de caso en la formoción del profesorado y la investigación didáctica, pp. 11-73. S. P. de la Universidad de Sevilla.

MELLADO, V. (1995a). Análisis del conocimiento didáctico del contenido, en profesores de ciencias de primaria $y$ secundaria en formación inicial. Cáceres: Servicio de Publicaciones de la Universidad de Extremadura.

MELLADO, V. (1995b). Concepciones de los profesores de ciencias en formación y práctica del aula, en Blanco, L. y Mellado, V. (eds.), La Formación del profesorado deciencias. y matemáticas en España y Portugal, pp. 309-326. Badajoz: Diputación Provincial. 
MELLADO, V. y BERMFJO, M.L. (1995). Los diarios de prácticas en la formación de maestros, Revista Interuniversitaria de Formación del Profesorado, 23, pp. 121-136.

MELLADO, V. y CARRACEDO, D. (1993). Contribuciones de la filosofía de la ciencia a la didáctica de las ciencias. Enseñanza de las Ciencias, 1)(3), pp. 331-339.

MITCHENER, C.P. y ANDERSON, R.D. (1989). Teachers' perspective: developing and implementing an STS curriculum. Journal of Research in Science Teaching, 26(4), pp. 351-369.

MORENO, A. (1995), Historia de la ciencia. Una posible contribución a la formación del profesorado, en Blanco, L y Mellado, V. (eds.), La formación del profesorado de ciencias y matemáticas en España y Portugal, pp. 345-360. Badajoz: Diputacion Provincial.

NEALE, D.C., SMITH, D.C. y WIER, E.A. (1987). Teacher thinking in elementary science instruction. Washington $\mathrm{DC}$ : AERA.

NOVAK, J.D. y GOWIN, D.B. (1988). Aprender a aprender. Barcelona: Martínez Roca.

ONTORIA, A. et aI. (1992). Mapas conceptuales. Una técnica para aprender. Madrid: Narcea.

POMEROY, D. (1993). Implications of teachers' beliefs about the nature of science: Comparison of the beliefs of scientist, secondary science teachers and elementary teachers. Science Education, 77(3), pp. 261-278

POPPER, K. (1983). Conjeturas y refutaciones. El desarrollo del pensamiento científico. Buenos Aires: Paidós.

PORLÁN, R. (1989). Teoría del conocimiento, teoria de la enseñanza y desarrollo profesional. Las concepciones epistemológicas de los profesores. Sevilia: Tesis doctoral inédita.

POWEL.L, R. (1994). From field science to classroom science: A case study constrained emergence in a second-career science teacher. Journal of Research in Science Teaching, 31(3), pp. 273-291

PRO, A. (1995). ¿Formación de profesores de secundaria vs. profesor tutor de pácticas de secundaria? en Blanco, L. y Mellado, V. (eds.), La formación del profesorado de ciencias y matemáticas en España y Portugal, pp. 375-398. Badajoz: Diputación Provincial.

RUBBA, P.A. y HARKNESS, W.L. (1993). Fxamination of preservice and inservice secondary science teachers' beliefs about science-technology-society interactions. Science Education, 77(4), pp. 407.431.

RUGGIERI, R., TARSITANI, C. y VICENTINI, M. (1993), The images of science of teachers in latin countries. International Joumal of Science Education, 15(4), pp. 383-393.

SCHON, D. (1983). The reflective practitioner. Nueva York: Basic Book.

SHAW, E.L.y CRONIN-JONES, L. (1989). Influence of methods, instructions on preservice elementary and secondary science teachers'beliefs. Paper presented at the Mid-South Ed. Research Ass. Little-Rock.

SHULMAN, L.S. (1986). Paradigms and Research programs in the study of teaching: A contemporary perspective. Version española de 1989. Paradigmas y programas de investigación en el estudio de la enseñanza: una perspectiva contemporánea, en Wittrock, La investigación de la enseñanza, l. Enfoq̨ues, teorias y métodos. Barcelona: Paidós.

SHULMAN, L.S. (1993). Renewing the pedagogy of teacher education: The impact of subject-specific conceptions of teaching, en Montero, M.L. y Vez, J.M. (eds.), Las didáticas específicas en la formación del profesorado, pp. 53-69. Santiago: Tórculo.

SMITH, D.C. y NEALE, D.C. (1991). The construction of subjet-matter knowledge in primary science teaching. Advances in Research on Teaching, 2, pp. 187-243.

SPEAR, M.G. (1984). Sex bias in science teachers' ratings of work and pupil characteristics, European Journal of Science Education, 6(4), pp. 369-377.

STODDART, T., CONNELL, M., STOFFE'TY, R. y PECK, D. (1993). Reconstructing elementary teacher cantidates' understanding of math and science content. Teaching \& Teacher Education, 9(3), pp. 229-241.

STODOLSKY, S.S. (1991). La importancia del contenido en la enseñanza. Actividades en las clases de matemáticas $y$ ciencias sociales. Madrid: MEC-Paidós

THOMAS, M.F. y GILBERT, I.K. (1989). A model for constructivist initial physics teacher education. International Journal of Science Education, 11(1), pp. 35-47.

TOBIN, K. (1993). Referents for making sense of science teaching. International Journal of science Education, 15(3), pp. $24 I-254$.

TOBIN, K. y ESPINET, M. (1989). Impediments to change: applications of coaching in high school science teaching. Journal of Research in Science Teaching, 26(2), pp. 105120 .

TOBIN, K., TIPPINS, D.J. y GALLARD, A.J. (1994). Research on Instructional Strategies for Teaching Science, en Gabel, D.L. (ed.), Handbook of Research on Science Teaching and Learning, pp. 45-93. Nueva York: Mcmillan PC.

VARFI,A, P. et al. (1993). Iniciación a la física en el marco de la teoria constructivista. Madrid: CIDE.

VILLAR, I.M. (1990). El profesorcomo profesional Fonnación y desarrollo personal. Granada: S.P. Universidad de Granada.

WALKER, R. (1983). La realización de estudios de casos en educación. Etica, teoría y procedimientos, en Dockrell y Ifamilton (eds.), Nuevas reflexiones sobre la investigución educativa, 42-82. Madrid: Narcea.

WALLACE, J. y I.OUDEN, W. (1992). Science teaching and teachers' knowledge: Prospect for reform of elementary classrooms. Science Education, 76(5), pp. 507-521.

WILSON, S.M., SHULMAN, L.S. y RICHERT, F.R. (1987), «150 differents ways» of knowing: Representations of knowledge in teaching, en Calderhead, I. (ed.), Exploring teachers' thinking. Nueva York: Taylor \& Francis.

WOODS, P. (1987). La escuela por dentro. La etnografía en la investigación educativa. Madrid: Paidós-MEC.

YOINNG, B.Y. y KELLOGG, T. (1993). Science attitudes and preparation of preservice clementary teachers. Science Education, 77(3), pp. 279-291. 